\title{
Problèmes écologiques posés par l'observation des Ascidies dans la zone infralittorale
}

\author{
Claude Monniot \\ Muséum National d'Histoire Naturelle, Brunoy, Essonne, France
}

\begin{abstract}
KURZFASSUNG: Ökologische Probleme, die sich aus Beobachtungen an Ascidien im Infralitoral ergeben. Die Verbreitung der Ascidien im Infralitoral kann nicht durch den Einfluß der im allgemeinen für die Gezeitenzone bedeutsamen ökologischen Faktoren erklärt werden, da diese im Infralitoral nur geringen Veränderungen unterliegen. Hinsichtlich des Vorkommens und der Verteilung der Ascidien läßt sich der Einfluß der Umwelt anhand folgender Kriterien erschließen: (a) Die relative Häufigkeit der Arten gibt einen Aufschluß über das Gleichgewicht zwischen günstigen und ungünstigen ökologischen Faktoren sowie über die Substratansprüche einer Species. (b) Der Einfluß ungünstiger Faktoren läßt sich anhand des Wachstums und des vorzeitigen Eintritts der Geschlechtsreife feststellen. (c) Die Anordnung auf dem Substrat ermöglicht die Beurteilung der Faktoren Licht, Farbe des Substrats und Orientierung im Verhältnis zum Substrat. Vergleichende Untersuchungen der Anordnung auf felsigem und sedimentärem Untergrund gestatten Aussagen über die Bedeutung der Bodenströmungen. Es wird auf die Notwendigkeit hingewiesen, moderne Unterwassergeräte und Tauchausrüstungen einzusetzen, um Untersuchungen über die "Aggregationsphänomene“ und eine Analyse der Lebensgemeinschaften in der infralitoralen Felszone sowie auf dem Meeresgrund durchführen zu können.
\end{abstract}

\section{INTRODUCTION}

Le développement de la plongée en scaphandre autonome, en soucoupe et en bathyscaphe a ouvert au biologiste un champ d'action considérable. Toutes les disciplines biologiques doivent et peuvent utiliser cette possibilité. Ce sont surtout le systématicien et l'écologiste qui sont les plus aptes à tirer un profit maximum de la plongée.

A Banyuls-sur-Mer et à Roscoff, des plongées régulières en scaphandre autonome m'ont permis de récolter et d'examiner un grand nombre d'Ascidies. Plusieurs espèces considérées comme très rares, ou même nouvelles, ont été trouvées grâce à la plongée. L'écologiste peut examiner de plus près, par ce procédé, la répartition des animaux sur le fond. Il met en évidence des facteurs écologiques rarement étudiés ou qui n'étaient même pas soupçonnés. 


\section{LES FACTEURS ÉCOLOGIQUES UTILISÉS DANS L'ÉTUDE DE LA ZONE LITTTORALE}

Le plongeur constate que dans la partie supérieure de la zone infralittorale, la mosaïque des populations est aussi diversifiée que dans la zone des marées. Mais, à partir d'une certaine profondeur, les conditions écologiques que l'on prend habituellement en considération sont beaucoup plus homogènes. On s'aperçoit au contraire que l'on ne peut expliquer la répartition des espèces par tous les critères simples, mesurables, envisagés dans la zone des marées: durée d'assèchement, température, salinité, $\mathrm{pH}, \mathrm{rH}$, turbulence. Ces caractéristiques deviennent pratiquement invariables dans l'infralittoral.

Dans le cas des Ascidies, la tolérance des espèces à ces facteurs écologiques, mesurée en aquarium, est plus grande que leur variation dans l'infralittoral. Nous sommes donc conduits à réviser nos méthodes et notre mode de pensée en pénétrant dans l'eau.

Trois critères principaux permettent de dire qu'une Ascidie est sensible à l'action d'un ou de plusieurs facteurs écologiques. Ce sont: (1) l'abondance relative de l'espèce, (2) sa taille et son état de maturité sexuelle, (3) sa disposition par rapport au substrat.

L'abondance relative de l'espèce donne une idée de la proportion des facteurs favorisants et des facteurs défavorables. Contrairement à ce qui se passe dans la zone littorale, il n'y a pratiquement jamais d'exclusion d'une espèce dans un milieu. Une espèce donnée est potentielle dans tout l'infralittoral.

Il faut alors tenir compte de l'abondance des surfaces utilisables par l'Ascidie, et de la fragmentation de ces surfaces. A Banyuls par exemple, à peu de distance et à une même profondeur, on trouve une majorité écrasante de Microcosmus sabatieri sur les rochers, alors que dans l'herbier à Posidonies on rencontrera surtout des $M$. polymorphus et $M$. nudistigma, pour lesquels une très faible surface solide est nécessaire à la fixation.

Dans le cas d'un substrat discontinu (ex. galets sur un fond vaseux), M. sabatieri est d'autant plus abondant que les galets sont plus nombreux. Si les galets sont dispersés on trouvera un ou deux individus sur $30 \%$ des galets. Si les galets sont proches la quasi-totalité de ceux-ci sera couverte de groupes de 5 à 10 Microcosmes.

La taille et la maturation sexuelle des Ascidies, considérées en même temps, représentent aussi un excellent indicateur. Dans les milieux favorables la croissance est rapide et l'activité génitale n'apparaît qu'à partir d'une taille déjà importante. En milieu défavorable les gonades sont déjà fonctionelles à une taille beaucoup plus réduite. Des malformations apparaissent souvent (d'après ce critère, pour beaucoup d'espèces, la zone littorale peut être considérée comme très défavorable).

La disposition par rapportausubstrat est peut-être ce qui renseigne le plus sur les besoins écologiques et le mode d'action de certains d'entre eux. C'est l'influence de la lumière qui est la plus évidente. Dans le fond, la lumière varie selon trois modalités liées au substrat: profondeur, orientation astronomique, orientation géodésique. A Banyuls $M$. sabatieri se rencontre à partir de 10 mètres sur les roches plates directement exposées à la lumière, à la même profondeur sur les parois verticales exposées au nord, et à $0 \mathrm{~m}$ sous de profonds surplombs. Certaines espèces telles que Pyura dura et Phallusia fumigata ne vivent que sous les surplombs. 
Par des expérimentations en aquarium Dybern \& Grave ont montré que certaines larves d'Ascidies sont sensibles à la couleur du substrat et à certaines longueurs d'ondes. Les études peuvent être effectuées assez facilement dans l'infralittoral. Ces facteurs jusqu'à présent méconnus doivent jouer un rôle non négligeable au niveau des associations biologiques.

\section{FACTEURS ECOLOGIQUES PROPRES Ã L'INFRALITTORAL}

Si la disposition des espèces dans la zone des marées est commandée presqu' uniquement par le facteur lumière, il en va tout autrement dans l'infralittoral où la disposition par rapport au substrat intervient, ce qui nous conduit à envisager l'action des facteurs écologiques propres à l'infralittoral.

Il existe des Ascidies sur certains fonds meubles. Dans de nombreux cas il s'agit d'espèces qui vivent aussi bien sur les fonds rocheux. On observe souvent une disposition précise de l'Ascidie sur le sédiment meuble, même quand sur la roche l'orientation est indifférente ou peu précisée. Plusieurs espèces du même genre peuvent prendre une orientation propre bien différente, par exemple Microcosmus sabatieri, M. vulgaris, ou les espèces du genre Ascidia. On observe à Roscoff par exemple les différences suivantes pour le genre Ascidia (Tableau 1).

\section{Tableau 1}

Comparaison des espèces du genre Ascidia sur les fonds rocheux et meubles

\begin{tabular}{|c|c|c|}
\hline Espèce & Fond rocheux & Fond meuble \\
\hline \multirow[t]{3}{*}{ Ascidia mentula } & fixée par la face gauche & $\begin{array}{l}\text { fixée seulement par la base de la } \\
\text { face gauche }\end{array}$ \\
\hline & $\begin{array}{l}\text { sous les rochers, dans les fentes } \\
\text { verticales }\end{array}$ & sur la face verticale d'un galet \\
\hline & $\begin{array}{l}\text { siphon buccal vers le haut seule- } \\
\text { ment si l'orientation du substrat } \\
\text { le permet }\end{array}$ & siphons toujours dressés \\
\hline \multirow[t]{4}{*}{ Ascidia virginea } & $\begin{array}{l}\text { fixée par toute la face gauche sur } \\
\text { les parois verticales }\end{array}$ & $\begin{array}{l}\text { fixée sur un galet ou sur une } \\
\text { coquille }\end{array}$ \\
\hline & $\begin{array}{l}\text { fixée par la partie postérieure sur } \\
\text { les parois horizontales ou obliques }\end{array}$ & \\
\hline & exceptionnelle sous les surplombs & \\
\hline & siphons toujours dressés & siphons toujours dressés \\
\hline \multirow[t]{2}{*}{ Ascidia conchylega } & fixée par toute la face gauche & $\begin{array}{l}\text { libre dans le sédiment aggloméré } \\
\text { les particules: sur la face gauche }\end{array}$ \\
\hline & $\begin{array}{l}\text { toujours sous les pierres, face } \\
\text { droite vers le bas }\end{array}$ & face droite dirigée vers le haut \\
\hline
\end{tabular}

Ces trois espèces peuvent vivre dans le même milieu. Leur abondance relative est en grande partie commandée par leurs exigences concernant la disposition. A la drague et par tous les moyens habituels de récolte, seule la portion des populations 
située sur les faces verticales et supérieures des enrochements sera prélevée. L'échantillonnage ainsi réalisé sera très différent de celui qui sera déterminé par le plongeur.

Pour expliquer la disposition de certaines Ascidies sur les fonds sédimentaires, il faut tenir compte de l'influence des courants sur ce fond. Le courant arrache à la pellicule superficielle du sédiment un nuage de quelques centimètres d'épaisseur. Ce nuage de suspensions contient aussi les particules tombées des couches supérieures, avec des particules sédimentaires fines et les nombreuses bactéries de l'interface eausédiment. Pour un organisme filtrant ou microphage, ce nuage a un triple effet: (1) abrasif, dû aux particules sableuses en suspension, effet qui peut être fondamental au moment de la fixation de la larve, (2) nutritif, par l'apport d'aliments tombés ou bactéries, (3) asphyxiant, par engorgement des appareils de capture des organismes microphages.

Mais la composition de ce nuage de particules est différente à la base et à son sommet par simple densité. L'analyse n'en a jamais été faite à notre connaissance. Son importance biologique est certainement fondamentale. L'étude de ce problème est à envisager en plongée.

La plupart des recherches effectuées sur les «associations biologiques» ont été poursuivies dans la zone des marées où les facteurs écologiques varient énormément et où le milieu apparaît comme une mosaïque très complexe. Plus les études progressent, plus on s'aperçoit cependant qu'il est difficile de trouver des espèces exclusives ou même préférentes, et cela malgré l'existence de conditions de vie extrêmement diversifiées. Dans l'infralittoral le problème est à la fois plus simple par la suppression de facteurs physiques variables, mais aussi plus complexe par l'interaction plus grande des individus entre eux.

Une association ne doit pas être comprise comme un groupe d'espèces contraint de vivre ensemble par des facteurs physicochimiques externes, mais comme des rassemblements spontanés se créant en milieu homogène.

Ce phénomène apparaît essentiel sur les fonds chalutables d'intérêt économique. Le "gregariousness phenomenon» doit être à la base d'un nouveau style d'associations, ainsi des taches d'espèces isolées sur le fond peuvent être subordonnées à d'autres. Par exemple, sur les fonds chalutables du golfe du Lion (Méditerranée), sur un fond homogène d'Alcyonnaires peuvent se superposer des taches d'Aglaophenia (Hydraires), de Microcosmus (Ascidies), ou de Pinna (Lamellibranches). A ces taches indépendantes mais imbriquées sont liées des faunes différentes forcément imbriquées elles-aussi. On aboutit ainsi à une association biologique complexe sur un milieu pourtant homogène. Ces associations évoluent en fonction du vieillissement de la population support. Mais si l'existence d'une des taches primitive est transitoire, l'ensemble de l'association représente un état beaucoup plus stable.

Ces phénomènes fondamentaux, ignorés par la drague ou le chalut, entrevus par le plongeur, ne peuvent être étudiés à fond que par des appareillages plus complexes: «Soucoupe plongeante», «Troika» télévision etc. A plus grande profondeur les bathyscaphes devront observer des phénomènes semblables, mais il est peu probable que la rentabilité puisse être immédiate, la faune des grands fonds étant encore trop peu connue.

La simple observation d'un groupe zoologique dans l'infralittoral permet de 
mettre en évidence l'influence d'un certain nombre de facteurs écologiques trop négligés. La plupart des plongeurs biologistes n'ont fait que transporter dans l'infralittoral les techniques d'étude employées dans la zone des marées. Ceci semble malheureusement insuffisant; il est nécessaire de créer de toute pièce une méthodologie de l'étude de l'infralittoral. A ce point de vue la création d'un laboratoire sous-marin immergé (type «maison sous la mer»), même dans des profondeurs faibles, rendrait des services considérables.

\section{RÉSUMÉ}

1. La répartition des Ascidies dans la zone infralittorale ne peut être expliquée par l'influence des facteurs écologiques généralement mesurés dans la zone des marées.

2. L'observateur dispose de trois critères principaux pour juger de l'action des facteurs écologiques non mesurables: (a) l'abondance relative des espèces renseigne sur la balance des facteurs favorables et défavorables ainsi que sur les besoins de surface de fixation; (b) l'état de croissance et la maturité sexuelle précoce permettent de détecter l'influence de facteurs défavorables; (c) la disposition par rapport au substrat permet de juger des facteurs lumière, couleur du substrat, orientation par rapport au substrat. L'étude comparée de la disposition sur fonds rocheux et sédimentaires permet de mettre en évidence l'importance du nuage particulaire soulevé par les courants de fond.

3. Scaphandre, Soucoupe et Troïka sont des instruments de choix pour l'analyse et l'expérimentation sur le «gregariousness phenomenon» et sur la formation des associations biologiques, dans l'infralittoral rocheux et plus encore sur les fonds chalutables.

\section{Discussion suivant le rapport de MONNIOT}

RIEDL: Die schönen Ergebnisse von Herrn Kollegen MonNiot sind ganz besonders erfreulich für einen Biologen, der ganz entsprechende Verhältnisse bereits bei anderen Tiergruppen, beispielsweise bei Hydroiden und Anthozoen, gesehen hat. Die hohen, vielfach radiären und langstieligen Formengruppen, welche die tieferen Sedimentgebiete selektiert haben, deuten ebenso darauf hin, daß es wichtig ist, den bei unruhiger See auftretenden Trübe-Schichten auszuweichen, um über denselben jene Bodenwasser-Schichten zu erreichen, in welchen Optimalbedingungen mit größerer Regelmäßigkeit realisiert sind.

Monniot: Certaines Ascidies telles Microcosmus vulgaris qui vit en Méditerranée à partir de $100 \mathrm{~m}$ de profondeur peuvent régler leur attitude en fonction de ce nuage particulaire. Si le nuage n'est pas trop dense, le plan d'ouverture du siphon est perpendiculaire à la direction du courant. Si le nuage est trop dense, ce plan s'oriente obliquement. 